(1)

CrossMark

\title{
More on the right ventricle in pulmonary hypertension
}

\author{
Robert Naeije ${ }^{1}$ and Stefano Ghio ${ }^{2}$ \\ Affiliations: ${ }^{1}$ Dept of Physiology, Erasme University Hospital, Brussels, Belgium. ${ }^{2}$ Dept of Cardiology, \\ Fondazione IRCCS Policlinico San Matteo, Pavia, Italy.
}

Correspondence: Robert Naeije, Dept of Physiology, Erasme Campus CP 604, 808 Lennik road, B-1070

Brussels, Belgium. E-mail: rnaeijedulb.ac.be

-

@ERSpublications

Decreased RVEF and PVR is associated with a decreased survival rate in PAH patients under targeted therapies http://ow.ly/EBK8U

In the present issue of the European Respiratory Journal, Courand et al. [1] report on the prognostic relevance of radionuclide angiography of the right ventricle (RV) in a cohort of 100 patients with idiopathic, heritable or drug-related pulmonary arterial hypertension (PAH). Patients with a baseline RV ejection fraction (RVEF) $>25 \%$ had better survival than those with a RVEF $<25 \%$. Furthermore, patients with a stable or increased RVEF at 3-6 months had a trend to better overall survival and a significantly lower cardiovascular mortality. These results are in keeping with recently demonstrated prognostic relevance of baseline and follow-up RVEF, but measured by magnetic resonance imaging (MRI) in PAH patients [2]. A striking finding in both studies was the dissociation between RVEF and pulmonary vascular resistance (PVR). Thus, a decreased PVR under targeted therapies could be associated with either deterioration or improvement in RV function $[1,2]$. The notion emerges that PAH is a disease of RVarterial uncoupling rather than only of pathological pulmonary vascular remodelling [3].

Right ventricular failure in pulmonary hypertension is the obvious consequence of prolonged exposure to excessive afterloading [3]. So, how is it possible that patients with a treatment-induced decrease in PVR might present with a worsening of RV function? The first possible answer is that the RV follows its own course of adaptation or maladaptation in increased pulmonary artery pressure (PAP), with considerable individual variation as a persistent, possibly genetic, biological mystery [4]. A second possible answer is in the inevitable systemic effects of pulmonary vasodilators. This has already been discussed at a time when $\mathrm{PAH}$ patients were tentatively treated with calcium channel blockers, hydralazine or minoxidil. All these systemic vasodilators increased cardiac output with no significant change in pulmonary vascular pressures, thus, decreased PVR. But associated increased systemic venous return would increase RV end-diastolic volume (EDV), increase RV afterload because of an increased wall tension (estimated by EDV $\times$ PAP) and decrease RVEF. SNiderman and Fitchett [5] called this the paradox of therapeutic success and clinical failure. High normal cardiac output with unchanged PAP may also be observed with targeted therapies, which thus have the potential of the unwelcome combination of decreased PVR and RVEF. It is not known how often this happens and how clinically relevant this maybe. In any case, CourNAND et al. [1] clearly demonstrate that one cannot be satisfied with PVR alone as a therapeutic goal in PAH.

The study by Courand et al. [1] is reminiscent of the flurry of reports of radionuclide angiographic measurements of RVEF in patients with chronic obstructive pulmonary disease (COPD), which were published in the 1970s and 1980s [6-12]. About half of these patients had a RVEF $<45-50 \%$, which was taken as the upper limit of normal [6-8]; a less stringent value than $30 \%$ considered by CourAND et al. [1]. One study reported failure of RVEF to rise during exercise, even in patients with a normal value at rest [9]. This was globally interpreted as "cor pulmonale" or RV failure on lung diseases and/or hypoxia. However, subsequent combined haemodynamic and radionuclide angiographic studies showed preserved 
systolic PAP to end-systolic volume (ESV) ratios [10], indicating preserved systolic function. RVEF was found to be more tightly correlated to fluid retention (on hypercapnia) rather to the severity of pulmonary hypertension [11]. RVEF predicted survival in COPD, but less significantly so than arterial blood gases or altered lung mechanics [12]. As correlations between RVEF and PAP or PVR were variable, sometimes tight [10] sometimes loose or even absent $[6,11]$, clinicians lost interest in radionuclide angiography of the RV for the diagnosis of cor pulmonale or detection of pulmonary hypertension.

Is there a brighter future for radionuclide RVEF in PAH? Probably not. Courand et al. [1] argue that radionuclide angiography is affordable and widely available, and that radiation exposure is not a concern except for repetitions in the youngest patients. However the procedure requires appointments at specialised departments, with possible waiting lists, and age-independent unequivocal safety of repetitions of the assessment of RV function is exactly what the follow-up of $\mathrm{PAH}$ requires. Furthermore, radionuclide angiography is a planar measure of a three-dimensional (3D) change and suffers from somewhat arbitrary automated contour definition. This explains variable and occasionally very low RVEF reported in normal subjects.

COURAND et al. [1] selected a RVEF of $25 \%$ as critical for the separation between good and bad prognosis. But this was a median value rather than a more methodologically correct sum of the highest specificity and sensitivity derived from receiver-operating characteristic curves, which would probably have yielded an even lower number. When RVEF was measured with MRI in similar PAH patients, a rigorously defined cut-off of $35 \%$ was found [2].

So, what would currently be the best and most practical way to evaluate RV function in PAH? Right heart catheterisation can measure stroke volume (SV) and right atrial pressure, but cannot measure RV volumes, and is, therefore, an imperfect surrogate for gold standard RV function definition by pressure-volume relationships [3, 13]. COURAND et al. [1] express disappointment about echocardiographic measurements such as M-mode tricuspid annular plane systolic excursion or tissue Doppler imaging of the maximum velocity of tricuspid annulus isovolumic contraction, both previously shown to be independent predictors of outcome in severe PH $[14,15]$. These turned out to be of little additional value to radionuclide RVEF [1]. However, modern echocardiography of the RV includes several further measurements of structure and function demonstrated to be of prognostic relevance [16] and nowadays preferably integrated in a multi-parametric evaluation $[17,18]$. Furthermore, developments in 3D-echocardiography open the perspective of accurate RV volume measurements [19]. Thus, echocardiographic measurements of RVEF are now possible, and have already been reported to be of prognostic relevance [20]. It has recently been suggested that a SV/ESV rather than SV/EDV, i.e. ejection fraction, may be a less preload-dependent estimate of RV function, and superior for the prognostication of patients with pulmonary hypertension [21]. This requires confirmation and can be further explored by bedside 3D-echocardiography.

Further added value of both MRI and echocardiography is insight into regional function and dyssynchrony (or inter-regional changes in strain and velocity of contraction) and asynchrony (or delaying of RV systole into left ventricular diastole), also called "post-systolic shortening" [22-24]. Regional RV function is efficiently explored by speckle tracking echocardiography, which evolves from 2D [25] to improved 3D [20] assessments. Speckle tracking echocardiography still requires time-consuming off-line analyses, but opens a fascinating perspective for further noninvasive and flexible research on RV function integrated into the daily practice of echocardiography [26].

The understanding of RV function in severe pulmonary hypertension is making rapid progress. Radionuclide angiography has delivered, but is now being replaced by MRI and 3D-echocardiography. In this context, Courand et al. [1] are to be commended for their enlightening contribution to the understanding of PAH as a RV failure syndrome.

\section{References}

1 Courand P-Y, Pina Jomir G, Khouatra C, et al. Prognostic value of right ventricular ejection fraction in pulmonary arterial hypertension. Eur Respir J 2015; 45: 139-149.

2 van de Veerdonk MC, Kind T, Marcus JT, et al. Progressive right ventricular dysfunction in patients with pulmonary arterial hypertension responding to therapy. J Am Coll Cardiol 2011; 58: 2511-2519.

3 Vonk-Noordegraaf A, Haddad F, Chin KM, et al. Right heart adaptation to pulmonary arterial hypertension: physiology and pathobiology. J Am Coll Cardiol 2013; 62: Suppl. 25, D22-D33.

4 Voelkel NF, Gomez-Arroyo J, Abbate A, et al. Mechanisms of right heart failure - a work in progress and plea for further prevention. Pulm Circ 2013; 3: 137-143.

5 Sniderman AD, Fitchett DH. Vasodilators and pulmonary arterial hypertension: the paradox of therapeutic success and clinical failure. Int J Cardiol 1988; 20: 173-181.

6 Ellis JH, Kirch D, Steele PP. Right ventricular ejection fraction in severe chronic airways obstruction. Chest 1977; 71: Suppl. 2, 281-282. 
7 Berger HJ, Matthay RA, Loke J, et al. Assessment of cardiac performance with quantitative radionuclide angiography: right ventricular ejection fraction with reference to findings in chronic obstructive pulmonary disease. Am J Cardiol 1978; 41: 897-905.

8 MacNee W, Xue QF, Hannan WJ, et al. Assessment by radionuclide angiography of right and left ventricular function in chronic bronchitis and emphysema. Thorax 1983; 38: 494-500.

9 Matthay RA, Berger HJ, Davies RA, et al. Right and left ventricular exercise performance in chronic obstructive pulmonary disease. Ann Intern Med 1980; 92: 234-239.

10 Brent BN, Berger HJ, Matthay RA, et al. Physiological correlates of right ventricular ejection fraction in chronic obstructive pulmonary disease: a combined radionuclide hemodynamic study. Am J Cardiol 1982; 50: 225-262.

11 MacNee W, Wathen C, Flenley D, et al. The effects of controlled oxygen therapy on ventricular function in patients with stable and decompensated cor pulmonale. Am Rev Respir Dis 1988; 137: 1289-1295.

12 France AJ, Prescott RJ, Biernacki W, et al. Does right ventricular function predict survival in patients with chronic obstructive pulmonary disease? Thorax 1988; 43: 621-626.

13 Vonk-Noordegraaf A, Westerhof N. Describing right ventricular function. Eur Respir J 2013; 41: 1419-1423.

14 Forfia PR, Fisher MR, Mathai SC, et al. Tricuspid annular displacement predicts survival in pulmonary hypertension. Am J Respir Crit Care Med 2006; 174: 1034-1041.

15 Ernande L, Cottin V, Leroux PY, et al. Right isovolumic contraction velocity predicts survival in pulmonary hypertension. J Am Soc Echocardiogr 2013; 26: 297-306.

16 Ghio S, Klersy C, Magrini G, et al. Prognostic relevance of the echocardiographic assessment of right ventricular function in patients with idiopathic pulmonary arterial hypertension. Int J Cardiol 2010; 140: 272-278.

17 Roberts JD, Forfia PR. Diagnosis and assessment of pulmonary vascular disease by Doppler echocardiography. Pulm Circ 2011; 1: 160-181.

18 Bossone E, D'Andrea A, D'Alto M, et al. Echocardiography in pulmonary arterial hypertension. J Am Soc Echocardiogr 2013; 26: 1-14.

19 Zhang QB, Sun JP, Gao RF, et al. Feasibility of single-beat full volume capture real-time three-dimensional echocardiography for quantification of right ventricular volume: validation by cardiac magnetic resonance imaging. Int J Cardiol 2013; 168: 3991-3995.

20 Smith BC, Dobson G, Dawson D, et al. Three-dimensional speckle tracking of the right ventricle: toward optimal quantification of right ventricular dysfunction in pulmonary hypertension. J Am Coll Cardiol 2014; 64: 41-51.

21 Vanderpool RR, Pinsky MR, Naeije R, et al. RV-pulmonary arterial coupling predicts outcome in patients referred for pulmonary hypertension. Heart 2014 [in press DOI: 10.1136/heartjnl-2014-306142].

22 Lopez-Candales A, Dohi K, Rajagopalan N, et al. Right ventricular dyssynchrony in patients with pulmonary hypertension is associated with disease severity and functional class. Cardiovasc Ultrasound 2005; 3: 23 .

23 Huez S, Faoro V, Vachiery JL, et al. Images in cardiovascular medicine. High-altitude-induced right-heart failure. Circulation 2007; 115: e308-e309.

24 Marcus JT, Gan CT, Zwanenburg JJ, et al. Interventricular mechanical asynchrony in pulmonary arterial hypertension: left-to-right delay in peak shortening is related to right ventricular overload and left ventricular underfilling. J Am Coll Cardiol 2008; 51: 750-757.

25 Meris A, Faletra F, Conca C, et al. Timing and magnitude of regional right ventricular function: a speckle tracking derived strain study of normal subjects and patients with right ventricular dysfunction. J Am Soc Echocardiogr 2010; 23: 823-831

26 Blanchard DG, DeMaria AN. Right ventricular 3-dimensional strain in pulmonary hypertension: the quest to see the future. J Am Coll Cardiol 2014; 64: 52-53. 